\title{
Regularity for evolution equations with non-autonomous perturbations in Banach spaces
}

Markus Penz

Citation: Journal of Mathematical Physics 59, 103512 (2018); doi: 10.1063/1.5011306

View online: https://doi.org/10.1063/1.5011306

View Table of Contents: http://aip.scitation.org/toc/jmp/59/10

Published by the American Institute of Physics

\section{Articles you may be interested in}

A maximum entropy method for solving the boundary value problem of second order ordinary differential equations

Journal of Mathematical Physics 59, 103505 (2018); 10.1063/1.5029856

On semiclassical Fourier integral operators, Schrödinger propagators and coherent states Journal of Mathematical Physics 59, 101504 (2018); 10.1063/1.5043363

Some multidimensional integrals in number theory and connections with the Painlevé $V$ equation Journal of Mathematical Physics 59, 091404 (2018); 10.1063/1.5038658

Nondegeneracy of the traveling lump solution to the $2+1$ Toda lattice Journal of Mathematical Physics 59, 101501 (2018); 10.1063/1.5038786

On Cauchy-Euler's differential equation involving a para-Grassmann variable Journal of Mathematical Physics 59, 103508 (2018); 10.1063/1.5047565

Quasi-periodic solution for the complex Ginzburg-Landau equation with continuous spectrum Journal of Mathematical Physics 59, 112701 (2018); 10.1063/1.5066453

\section{Don't let your writing keep you from getting published!}

\section{AIP $\mid$ Author Services}

Learn more today! 


\title{
Regularity for evolution equations with non-autonomous perturbations in Banach spaces
}

\author{
Markus Penz ${ }^{\text {a) }}$ \\ Max Planck Institute for the Structure and Dynamics of Matter, Hamburg, Germany
}

(Received 31 October 2017; accepted 12 October 2018; published online 31 October 2018)

\begin{abstract}
We provide regularity of solutions to a large class of evolution equations on Banach spaces where the generator is composed of a static principal part plus a nonautonomous perturbation. Regularity is examined with respect to the graph norm of the iterations of the principal part. The results are applied to the Schrödinger equation and conditions on a time-dependent scalar potential for the regularity of the solution in higher Sobolev spaces are derived. Published by AIP Publishing. https://doi.org/10.1063/1.5011306
\end{abstract}

\section{INTRODUCTION}

In this work, we study the existence and regularity of solutions to the evolution equation

$$
\partial_{t} u(t)=G(t) u(t) \quad \text { with } \quad G(t)=A+B(t)
$$

for finite times $t \in[0, T]$ in a Banach space $X$. The generator $G(t)$ is composed of a principal static part $A$ and a potentially time-dependent (non-autonomous) perturbation $B(t)$. The conditions for existence of an evolution system (solution operator) $U(t, s)$ are formulated with respect to the domain $D(A)$ of the principal part. Since $A$ is assumed closed, this domain is a Banach space in itself if equipped with the graph norm $\|x\|_{D(A)}=\|x\|_{X}+\|A x\|_{X}$. In fact we will show with Theorem IV.4 that if the generator belongs to a (quasi)contraction semigroup and $B$ fulfils higher-order relative boundedness with respect to $A$ (Definition III.1) and a Lipschitz property (III.4), then $U(t, s)$ is a bounded operator $D\left(A^{m}\right) \rightarrow D\left(A^{m}\right)$. This stands in contrast to approaches that study regularity in pre-defined classes, whereas here the respective regularity class is directly yielded by the generator's principal part.

This result in the setting of an $N$-particle Hilbert space $X=L^{2}\left(\Omega^{N}\right)$ and Schrödinger-type equations leads to the regularity of propagated wave functions in terms of the graph norm of the iterated Laplacian. The perturbation $B(t)$ can be considered as consisting of external or inter-particle potentials of a certain Kato-perturbation type (Definition V.7). Since the graph norm of $D\left(\Delta^{m}\right)$ is equivalent to the usual Sobolev space norm, an important result considering the regularity of Schrödinger solutions with respect to Sobolev spaces is achieved (see final Theorem V.9). The growth estimate for the Sobolev norm over time is of exponential type (38). In the case of potentials that can be assumed smooth in space and time and periodic in space, notable results concerning the (linear) growth of Sobolev norms have been achieved by Bourgain (1999) and more recently by Delort (2010). A very nice recent work aimed at studying the time regularity of solutions to facilitate the Runge-Gross proof of time-dependent density functional theory (Ruggenthaler-Penz-van Leeuwen, 2015) can be found in Fournais et al. (2016).

The existence part of the proof of our main Theorem IV.4 is similar to the original treatment of Kato (1953), the usual reference point is Reed-Simon II (1975, Theorem X.70), but we give more general conditions that are also easier to check. This is in the spirit of a recent effort by Schmid and Griesemer (2014) to simplify and standardise the classical existence results for (1) to the simple condition that $t \mapsto G(t) x$ is continuously differentiable for all $x \in D(G)$. This was followed in Schmid and

\footnotetext{
a) Author to whom correspondence should be addressed: markus.penz@mpsd.mpg.de
} 
Griesemer (2016) by a generalization to Lipschitz continuity which already bears some similarities to this work but includes no higher regularity estimates. Furthermore Schmid and Griesemer (2016) built their argument around uniformly convex spaces which is, to our understanding, not necessary, since the more general notion of reflexivity is sufficient. Reflexivity is especially convenient because it is conserved when switching to equivalent norms, which is heavily used throughout this work, while uniform convexity is not.

A more comprehensive version of this article that includes detailed explanations, additional proofs, and further references can be found as a preprint at arXiv:1801.03361.

\section{GRAPH-NORM SPACES}

The setting is always a Banach space $X$ with norm $\|x\|=\|x\|_{X}$. The domain and range (image) of an operator will be denoted as $D(A)$ and $R(A)$, respectively. We define the graph norm for a linear operator $A: X \rightarrow X$, generally unbounded, and $x \in D(A)$ as $\|x\|_{D(A)}=\|x\|+\|A x\|$. This definition yields an equivalent norm to the more frequently given expression for the graph norm as a Pythagorean sum. The inequality

$$
\sqrt{\|x\|^{2}+\|A x\|^{2}} \leq\|x\|+\|A x\| \leq \sqrt{2} \sqrt{\|x\|^{2}+\|A x\|^{2}}
$$

is easily shown to hold by squaring it and using the inequality of arithmetic and geometric means, i.e., $\|x\|\|A x\| \leq \frac{1}{2}\left(\|x\|^{2}+\|A x\|^{2}\right)$. The symbol “ " will later be used to denote equivalence of norms.

The domain $D\left(A^{k}\right)$ of the iterated operator is the set of all Banach space elements $x \in X$ where for all $1 \leq j \leq k$ also $A^{j} x \in X$ holds. [See Fournais et al. (2016) for a special emphasis on this in the context of studying the time-regularity of solutions to the Schrödinger equation with Coulomb potentials.] Note that $D\left(A^{0}\right)=D(I)=X$. Since the main tool of analysis will be higher-order graph-norm spaces $D\left(A^{k}\right)$, we adopt a shorthand notation for their norms and the respective operator norms,

$$
\begin{gathered}
\|x\|_{(k)}=\|x\|_{D\left(A^{k}\right)}=\|x\|+\|A x\|+\cdots+\left\|A^{k} x\right\|, \\
\|T\|_{(k, l)}=\|T\|_{\mathcal{B}\left(D\left(A^{k}\right), D\left(A^{l}\right)\right)}=\sup _{\substack{x \in D\left(A_{k}\right) \\
x \neq 0}} \frac{\|T x\|_{(l)}}{\|x\|_{(k)}} .
\end{gathered}
$$

The parentheses in the subscript shall discern this notation from the usual $L^{p}$ and $W^{k, p}$ norms $\|\cdot\|_{p}$ and $\|\cdot\|_{k, p}$. We directly note the following chain of continuous embeddings:

$$
D\left(A^{k}\right) \hookrightarrow D\left(A^{k-1}\right) \hookrightarrow \ldots \hookrightarrow D(A) \hookrightarrow X .
$$

In correspondence with spaces equipped with the graph norm the notion of closed operators is allimportant because $D(A)$ is a Banach space if and only if $A$ is closed. A closed operator is clearly always bounded as $A: D(A) \rightarrow X$ because of $\|A x\| \leq\|x\|_{(1)}$.

Lemma II.1. If $A$ is closed with a non-empty resolvent set then all its iterations $A^{k}, k \in \mathbb{N}$, are closed.

Proof. Take $\lambda \in \rho(A)$, then the iterated resolvent operator $(A-\lambda I)^{-k}$ is everywhere defined and bounded and thus closed (closed graph theorem). Since a closed, invertible operator has a closed inverse [Engel and Nagel, 2000, Definition B.1(e)], we also have $(A-\lambda I)^{k}$ closed. This expression expands to

$$
(A-\lambda I)^{k}=\sum_{j=0}^{k}\left(\begin{array}{l}
k \\
j
\end{array}\right)(-\lambda)^{k-j} A^{j},
$$

which facilitates an easy induction scheme. From $I, A,(A-\lambda I)^{2}$ closed, we arrive at $A^{2}$ closed and so on.

Lemma II.2. For A: $X \rightarrow X$ closed, $D(A)$ equipped with the graph norm is a reflexive Banach space if $X$ is so. 
Proof. Take the isometry

$$
i: D(A) \longrightarrow X \times X, x \longmapsto(x, A x),
$$

where for $x \in D(A)$ we have the usual graph norm $\|x\|+\|A x\|$ and for $\left(x_{1}, x_{2}\right) \in X \times X$ the sum norm $\left\|\left(x_{1}, x_{2}\right)\right\|=\left\|x_{1}\right\|+\left\|x_{2}\right\|$. Now $i(D(A))$ is closed in $X \times X$ as the image of a closed set under an isometry and $X \times X$ is known to be reflexive if $X$ is. But any closed subspace of a reflexive space if reflexive itself, so $D(A)$ is.

\section{RELATIVE BOUNDEDNESS AND A PREPARATORY LEMMA}

A final preparatory lemma will help us to establish the desired regularity result in Sec. IV and gives the main condition on the non-autonomous perturbation $B(t)$ in the form of higher-order relative boundedness with respect to $A$ and a Lipschitz property. The assumption that all $A^{k}$ are closed is imperative to have Banach spaces $D\left(A^{k}\right)$. In our main Theorem IV.4, we will then not have this condition explicitly, but rely on Lemma II.1 to get closedness. We now define the new notion of $A$ boundedness of order $m$, a generalization of the usual A-boundedness [Reed and Simon II (1975, X.2) and Kato (1995, IV.1.1)] that corresponds to order $k=1$.

Definition III.1. Let A, B be densely defined operators and A closed, then B is called A-bounded of order $\boldsymbol{m}$ if

(i) $D(A) \subseteq D(B)$ and

(ii) There are $a, b \geq 0$ such that for all $1 \leq k \leq m$ and $x \in D\left(A^{k}\right)$, it holds that $\|B x\|_{(k-1)} \leq a\left\|A^{k} x\right\|$ $+b\|x\|_{(k-1)}$.

The infimum of possible values for a is called the relative bound (of order $m$ ) of $B$ with respect to $A$. The relative bound can be as low as 0 , the operator is then called infinitesimally small.

Note III.2. From this definition, it directly follows that if $B$ is $A$-bounded of order $m$, then for all $0 \leq k \leq m$ we have $B: D\left(A^{k}\right) \rightarrow D\left(A^{k-1}\right)$ bounded with respect to the $\|\cdot\|_{(k, k-1)}$ operator norm. Note further that the usual $A$-boundedness of $B$ with relative bound strictly smaller than 1 also means that $A+B$ is closed if and only if $A$ is. (Kato, 1995, Theorem IV.1.1) Another very similar application of relative boundedness is the famous Kato-Rellich theorem that enters Theorem V.2 here. A further consequence expressed in the following lemma is that the spaces $D\left(A^{k}\right)$ and $D\left(G^{k}\right)$, where $G=A+B$, become equivalent.

Lemma III.3. Assume A closed with non-empty resolvent set and $B$ to be A-bounded of order $m$ with relative bound $<1$. Then for $G=A+B$, it holds $D\left(G^{k}\right)=D\left(A^{k}\right)$ and the respective graph norms are equivalent for all $1 \leq k \leq m$.

Proof. We start with $k=1$ and assume $x \in D(A)$, then $A$-boundedness yields

$$
\|G x\| \leq\|A x\|+\|B x\| \leq(1+a)\|A x\|+b\|x\|
$$

and thus $x \in D(G)$. On the other hand, assume $x \in D(G)$, then one has from $A=G-B$

$$
\|A x\| \leq\|G x\|+\|B x\| \leq\|G x\|+a\|A x\|+b\|x\|
$$

and because of $a<1$ by assumption

$$
\|A x\| \leq \frac{1}{1-a}\|G x\|+\frac{b}{1-a}\|x\|
$$

and thus $x \in D(A)$. This establishes $D(G)=D(A)$ and further by the inequalities (7) and (9) the equivalence of the norms.

Next we proceed by induction and assume that the argument holds for $k-1$. Let $x \in D\left(A^{k}\right)$, then

$$
\begin{aligned}
\|G x\|_{(k-1)} & \leq\|A x\|_{(k-1)}+\|B x\|_{(k-1)} \leq\|A x\|_{(k-1)}+a\left\|A^{k} x\right\|+b\|x\|_{(k-1)} \\
& \leq(1+a+b)\|x\|_{(k)} .
\end{aligned}
$$


Since we already established $D\left(G^{k-1}\right)=D\left(A^{k-1}\right)$ with equivalent norms, the expression $\|G x\|_{(k-1)}+\|x\|$ corresponds to the graph norm of $D\left(G^{k}\right)$ (up to a multiplicative constant). On the other hand, let $x \in D\left(G^{k}\right)$ then

$$
\begin{aligned}
\left\|A^{k} x\right\|+\|x\|_{(k-1)}-\|x\|=\|A x\|_{(k-1)} & \leq\|G x\|_{(k-1)}+\|B x\|_{(k-1)} \\
& \leq\|G x\|_{(k-1)}+a\left\|A^{k} x\right\|+b\|x\|_{(k-1)}
\end{aligned}
$$

and after a simple manipulation

$$
(1-a)\|x\|_{(k)} \leq(1-a)\left\|A^{k} x\right\|+\|x\|_{(k-1)} \leq\|G x\|_{(k-1)}+\|x\|+b\|x\|_{(k-1)} .
$$

The right-hand side corresponds again to the graph norm of $D\left(G^{k}\right)$ and thus by inequalities $(10)$ and (12), we get $D\left(G^{k}\right)=D\left(A^{k}\right)$ and the equivalence of the respective norms.

Lemma III.4. Let $G(t)=A+B(t)$ have $0 \in \rho(G(t))$ at all times under consideration. Furthermore assume $A^{k}$ to be closed for all $1 \leq k \leq m, B(t)$ to always be A-bounded of order $m$ with a maximal relative bound strictly smaller than 1 , and demand the Lipschitz condition

$$
L_{m}:=\sup _{t^{\prime} \neq t} \sum_{k=1}^{m} \frac{\left\|B\left(t^{\prime}\right)-B(t)\right\|_{(k, k-1)}}{\left|t^{\prime}-t\right|}<\infty .
$$

Then $G\left(t^{\prime}\right)^{m} G(t)^{-m}=K_{m}\left(t^{\prime}, t\right)+I$ with the operator $K_{m}\left(t^{\prime}, t\right): X \rightarrow X$ bounded by $C_{m} L_{m}\left|t^{\prime}-t\right|$ if $t^{\prime} \neq t$. [See (21) for a definition of the G-dependent constants $\left.C_{m}.\right]$

Proof. In the whole proof, we mostly write $G=G(t), G^{\prime}=G\left(t^{\prime}\right)$ and similarly for $B$ for brevity. For all $1 \leq k \leq m$ clearly $A: D\left(A^{k}\right) \rightarrow D\left(A^{k-1}\right)$ bounded by definition and $B$ bounded equally by assumption (Note III.2). This makes the combined operator $G: D\left(A^{k}\right) \rightarrow D\left(A^{k-1}\right)$ bounded as well. Because of zero in the resolvent set, we have a well-defined and bounded $G^{-1}: X \rightarrow X$. We continue with the restriction of $G^{-1}$ on $D\left(A^{k-1}\right)$ that will still be denoted $G^{-1}$. Because $B$ is $A$-bounded of order $m$, we get for all $x \in D\left(A^{k-1}\right)=D\left(G^{k-1}\right), G^{-1} x \in D\left(G^{k}\right)=D\left(A^{k}\right)$ (Lemma III.3)

$$
\begin{aligned}
\left\|A^{k} G^{-1} x\right\| & =\left\|A^{k-1}(G-B) G^{-1} x\right\| \\
& \leq\left\|B G^{-1} x\right\|_{(k-1)}+\|x\|_{(k-1)} \\
& \leq a\left\|A^{k} G^{-1} x\right\|+b\left\|G^{-1} x\right\|_{(k-1)}+\|x\|_{(k-1)}
\end{aligned}
$$

and since $a<1$ it holds

$$
\left\|A^{k} G^{-1} x\right\| \leq \frac{b}{1-a}\left\|G^{-1} x\right\|_{(k-1)}+\frac{1}{1-a}\|x\|_{(k-1)} .
$$

This result establishes an estimate

$$
\begin{aligned}
\left\|G^{-1} x\right\|_{(k)} & =\left\|G^{-1} x\right\|_{(k-1)}+\left\|A^{k} G^{-1} x\right\| \\
& \leq\left(\frac{b}{1-a}+1\right)\left\|G^{-1} x\right\|_{(k-1)}+\frac{1}{1-a}\|x\|_{(k-1)}
\end{aligned}
$$

and after $k-1$ further iterations yields $G^{-1}: D\left(A^{k-1}\right) \rightarrow D\left(A^{k}\right)$ bounded. The iterated operators $G^{k}: D\left(A^{k}\right) \rightarrow X, G^{-k}: X \rightarrow D\left(A^{k}\right)$ are thus bounded as well, just like $K_{k}\left(t^{\prime}, t\right): X \rightarrow X$ defined by $K_{k}\left(t^{\prime}, t\right):=G\left(t^{\prime}\right)^{k} G(t)^{-k}-I$. We still have to show the special bound for $K_{m}$. We start with $k=1$

$$
K_{1}=G^{\prime} G^{-1}-I=\left(G^{\prime}-G\right) G^{-1}=\left(B^{\prime}-B\right) G^{-1} .
$$

The operator $K_{1}$ has the bound

$$
\left\|K_{1}\right\|_{(0,0)}=\left\|\left(B^{\prime}-B\right) G^{-1}\right\|_{(0,0)} \leq\left\|B^{\prime}-B\right\|_{(1,0)}\left\|G^{-1}\right\|_{(0,1)} .
$$

Now from $k=1$, on we proceed by iteration,

$$
K_{k}=G^{\prime k} G^{-k}-I=G^{\prime k-1}\left(G^{\prime}-G\right) G^{-k}+K_{k-1}=G^{\prime k-1}\left(B^{\prime}-B\right) G^{-k}+K_{k-1} .
$$

The estimate is now 


$$
\begin{aligned}
\left\|K_{k}\right\|_{(0,0)} & \leq\left\|G^{\prime k-1}\left(B^{\prime}-B\right) G^{-k}\right\|_{(0,0)}+\left\|K_{k-1}\right\|_{(0,0)} \\
& \leq\left\|G^{\prime k-1}\right\|_{(k-1,0)}\left\|B^{\prime}-B\right\|_{(k, k-1)}\left\|G^{-k}\right\|_{(0, k)}+\left\|K_{k-1}\right\|_{(0,0)},
\end{aligned}
$$

which sums up to

$$
\begin{aligned}
\left\|K_{m}\right\|_{(0,0)} & \leq \sum_{k=1}^{m}\left\|G^{\prime k-1}\right\|_{(k-1,0)}\left\|B^{\prime}-B\right\|_{(k, k-1)}\left\|G^{-k}\right\|_{(0, k)} \\
& \leq \max _{1 \leq k \leq m}\left\{\left\|G^{\prime k-1}\right\|_{(k-1,0)}\left\|G^{-k}\right\|_{(0, k)}\right\} \times \sum_{k=1}^{m} \frac{\left\|B^{\prime}-B\right\|_{(k, k-1)}}{\left|t^{\prime}-t\right|} \times\left|t^{\prime}-t\right| \\
& \leq \underbrace{\sup _{t^{\prime} \neq t} \max _{1 \leq k \leq m}\left\{\left\|G^{\prime k-1}\right\|_{(k-1,0)}\left\|G^{-k}\right\|_{(0, k)}\right\}}_{C_{m}} \times \underbrace{\sup _{t^{\prime} \neq t} \sum_{k=1}^{m} \frac{\left\|B^{\prime}-B\right\|_{(k, k-1)}}{\left|t^{\prime}-t\right|}}_{L_{m}} \times\left|t^{\prime}-t\right| .
\end{aligned}
$$

\section{REGULARITY RESULT}

First we will stick to a pure Banach space setting even though the strongest motivation is of course the Schrödinger equation that will be discussed in Sec. V. The domain of the time-dependent generator $G(t)$ is usually assumed to remain constant in time and we write $D(G(t))=D(G)$. This is true anyway by assumption following Lemma III.4 where $D(G(t))=D(A)$ always holds.

Definition IV.1. An evolution system belonging to an evolution equation like (1) is a twoparameter family of bounded linear operators $U(t, s), 0 \leq s \leq t \leq T$, on X that fulfils (Pazy, 1983, Chap. 5, Definition 5.3)

(i) $U(s, s)=I, U(t, r) U(r, s)=U(t, s)$ for all $0 \leq s \leq r \leq t \leq T$,

(ii) $(t, s) \mapsto U(t, s)$ is jointly strongly continuous, i.e., $\lim _{t \rightarrow s} U(t, s)=I$ strongly and equivalently for $s \rightarrow t$,

and on $D(G)$ solves

$$
\begin{aligned}
& \partial_{t} U(t, s)=G(t) U(t, s), \\
& \partial_{s} U(t, s)=-U(t, s) G(s) .
\end{aligned}
$$

Note IV.2. In the Hilbert space setting with $H(t)=\mathrm{i} G(t)$ self-adjoint (i.e., $G(t)$ is skew-adjoint) that will be discussed in Sec. V, the unitarity condition $U(t, s)^{*}=U(t, s)^{-1}=U(s, t)$ is added to the properties of the evolution system.

Note IV.3. In the case of a time-independent generator $G$, the typical setting is that of a strongly continuous one-parameter semigroup (Renardy and Rogers, 2004; Engel and Nagel, 2000; and Pazy, 1983). The generator of a strongly continuous semigroup is always densely defined and closed (Renardy and Rogers, 2004, Theorem 12.12). A quasicontraction semigroup $U(t)$ is a strongly continuous semigroup with a constant $\omega>0$ such that for all $x \in X$ and $t \geq 0$

$$
\|U(t) x\| \leq \mathrm{e}^{\omega t}\|x\| .
$$

Clearly in the Hilbert space setting with a unitary (semi-)group, it always holds $\|U(t) x\| \leq\|x\|$ which makes $U(t)$ trivially a contraction semigroup with $\omega=0$. The generator of a quasicontraction semigroup is known to have all $\lambda \in \mathbb{C}$ with $\operatorname{Re} \lambda>\omega$ in the resolvent set as a corollary to the famous Hille-Yosida generation theorem (Engel and Nagel, 2000, Corollary II.3.6). Thus $G$ as such a generator just needs to be shifted by $\omega+1$ to have $0 \in \rho(G)$ as demanded by Lemma III.4. What follows is our main result regarding the regularity property of evolution systems.

Theorem IV.4. On $X$ a reflexive Banach space, let $G(t)=A+B(t)$ be the generator of a quasicontraction semigroup for all $t \in[0, T]$ with a common contraction constant $\omega$, let $A$ be closed 
with a non-empty resolvent set, and let B have the properties of Lemma III.4. Then the evolution Eq. (1) has a well-defined evolution system given by the limit of the stepwise static approximation (24) that is bounded $D\left(A^{k}\right) \rightarrow D\left(A^{k}\right)$ for all $0 \leq k \leq m$ and thus preserves regularity of Banach space vectors in the class $D\left(A^{m}\right)$.

Proof. First note that because of $A$ closed with non-empty resolvent set we have by Lemma II.1 all $A^{k}$ closed and thus can work in Banach spaces $D\left(A^{k}\right)$.

Let $P_{k}$ be a sequence of equidistant partitions of $[0, T]$ with $k$ subintervals $\left[t_{i}, t_{i+1}\right]$ with $t_{0}=0$, $t_{k}=T$, and mesh size $T / k$ going to zero as $k \rightarrow \infty$. We write $\lfloor s\rfloor_{k}$ for the largest $t_{i}$ in the partition $P_{k}$ smaller or equal than $s \in[0, T]$. We define the stepwise static approximation to the evolution system by combining the $k$ individual evolution semigroups $U_{k}^{(i)}$ defined by the static generators $G\left(t_{i}\right), 0 \leq i \leq k-1$

$$
\begin{aligned}
& U_{k}(t, s)=U_{k}^{(i)}(t-s) \quad \text { if } t_{i} \leq s \leq t \leq t_{i+1}, \\
& U_{k}(t, s)=U_{k}\left(t, t_{i}\right) U_{k}\left(t_{i}, s\right) \quad \text { with } s<t_{i}<t \text { else. }
\end{aligned}
$$

We show now that for $k \rightarrow \infty$, the $U_{k}(t, s)$ converges uniformly in $t$ and preserves the desired degree of regularity. Convergence is tested with the Cauchy property of the sequence $U_{k}$. We use $\partial_{t} U_{k}(t, s)$ $=G\left(\lfloor t\rfloor_{k}\right) U_{k}(t, s)$ and $\partial_{s} U_{k}(t, s)=-U_{k}(t, s) G\left(\lfloor s\rfloor_{k}\right)$ which follows directly from the definition of $U_{k}$ above and the evolution semigroup property of $U_{k}{ }^{(i)}$

$$
\begin{aligned}
U_{k}(t, s)-U_{l}(t, s) & =\left.U_{l}(t, r) U_{k}(r, s)\right|_{r=s} ^{t} \\
& =\int_{s}^{t} \partial_{r}\left(U_{l}(t, r) U_{k}(r, s)\right) \mathrm{d} r \\
& =\int_{s}^{t}\left(\left(\partial_{r} U_{l}(t, r)\right) U_{k}(r, s)+U_{l}(t, r)\left(\partial_{r} U_{k}(r, s)\right)\right) \mathrm{d} r \\
& =-\int_{s}^{t} U_{l}(t, r)\left(G\left(\lfloor r\rfloor_{l}\right)-G\left(\lfloor r\rfloor_{k}\right)\right) U_{k}(r, s) \mathrm{d} r \\
& =-\int_{s}^{t} U_{l}(t, r)\left(B\left(\lfloor r\rfloor_{l}\right)-B\left(\lfloor r\rfloor_{k}\right)\right) U_{k}(r, s) \mathrm{d} r .
\end{aligned}
$$

(Note that a problem arises with the time derivative if $r=t_{i}$ because then the right and left derivatives do not match. But this is just at a finite number of points that can always be omitted from the integral.) We check the Cauchy property in the $\|\cdot\|_{(m, m-1)}$ norm

$$
\left\|U_{k}(t, s)-U_{l}(t, s)\right\|_{(m, m-1)} \leq \int_{s}^{t}\left\|U_{l}(t, r)\right\|_{(m-1, m-1)}\left\|B\left(\lfloor r\rfloor_{l}\right)-B\left(\lfloor r\rfloor_{k}\right)\right\|_{(m, m-1)}\left\|U_{k}(r, s)\right\|_{(m, m)} \mathrm{d} r .
$$

Since $B(t)$ has the Lipschitz-property up to order $m$ which implies continuity in the $\mathcal{B}\left(D\left(A^{m}\right), D\left(A^{m-1}\right)\right)$ norm, the difference would go to zero if $k \rightarrow \infty$. But are $\left\|U_{l}(t, r)\right\|_{(m-1, m-1)}$ and $\left\|U_{k}(r, s)\right\|_{(m, m)}$ uniformly bounded? We will test for $\left\|U_{k}(r, s)\right\|_{(m, m)}<\infty$, all lower orders at all times $0 \geq s \geq r \geq T$ applied equally.

The idea is to switch to a shifted, auxiliary generator $\tilde{G}(t)=G(t)-(\omega+1)$ such that $0 \in \rho(\tilde{G}(t))$ for all $t$ (also see Note IV.3) thus achieving accordance with the conditions of Lemma III.4. Then we introduce the identities $\tilde{G}^{-m}\left(t_{i}\right) \tilde{G}^{m}\left(t_{i}\right)$ in front of all the short-time evolution operators, exchange them with the evolution operators originating from the same generator, and give an estimate for the arising terms involving $K_{m}$ by Lemma III.4 applied to $\tilde{G}$. Let $i$ be such that $\lfloor s\rfloor_{k}=t_{i}$, i.e., $i=\lfloor s k / T\rfloor$ (usual floor function brackets), and $j$ be such that $\lfloor r\rfloor_{k}=t_{j}$, i.e., $j=\lfloor r k / T\rfloor$

$$
\begin{aligned}
U_{k}(r, s)= & U_{k}^{(j)}\left(r-t_{j}\right) U_{k}^{(j-1)}\left(t_{j}-t_{j-1}\right) \ldots U_{k}^{(i)}\left(t_{i+1}-s\right) \\
= & \tilde{G}^{-m}\left(t_{j}\right) U_{k}^{(j)}\left(r-t_{j}\right) \tilde{G}^{m}\left(t_{j}\right) \tilde{G}^{-m}\left(t_{j-1}\right) U_{k}^{(j-1)}\left(t_{j}-t_{j-1}\right) \tilde{G}^{m}\left(t_{j-1}\right) \ldots \\
& \ldots \tilde{G}^{-m}\left(t_{i+1}\right) U_{k}^{(i+1)}\left(t_{i+2}-t_{i+1}\right) \tilde{G}^{m}\left(t_{i+1}\right) \tilde{G}^{-m}\left(t_{i}\right) U_{k}^{(i)}\left(t_{i+1}-s\right) \tilde{G}^{m}\left(t_{i}\right) .
\end{aligned}
$$


We write the $\tilde{G}\left(t^{\prime}\right)^{m} \tilde{G}(t)^{-m}$ encounters like in Lemma III.4 as $K_{m}\left(t^{\prime}, t\right)+I$

$$
\begin{gathered}
U_{k}(r, s)=\tilde{G}^{-m}\left(t_{j}\right) U_{k}^{(j)}\left(r-t_{j}\right) \prod_{l=i+1}^{j-1}\left(K_{m}\left(t_{l+1}, t_{l}\right)+I\right) U_{k}^{(l)}\left(t_{l+1}-t_{l}\right) \\
\left(K_{m}\left(t_{i+1}, t_{i}\right)+I\right) U_{k}^{(i)}\left(t_{i+1}-s\right) \tilde{G}^{m}\left(t_{i}\right) .
\end{gathered}
$$

(Note that the product is time-ordered.) Next we estimate the $\|\cdot\|_{(m, m)}$ norm of this expression using the result from Lemma III.4 and the regular mesh size $t_{i}-t_{i-1}=T / k$. For this, we repeatedly use the quasicontraction property that assures $\left\|U_{k}^{(l)}\left(t_{l+1}-t_{l}\right)\right\|_{(0,0)} \leq \mathrm{e}^{\omega\left(t_{l+1}-t_{l}\right)}$.

$$
\begin{aligned}
\left\|U_{k}(r, s)\right\|_{(m, m)} & \leq \mathrm{e}^{\omega(r-s)}\left\|\tilde{G}^{-m}\left(t_{j}\right)\right\|_{(0, m)} \prod_{l=i}^{j-1}\left(\left\|K_{m}\left(t_{l+1}, t_{l}\right)\right\|_{(0,0)}+1\right)\left\|\tilde{G}^{m}\left(t_{i}\right)\right\|_{(m, 0)} \\
& \leq \mathrm{e}^{\omega(r-s)}\left\|\tilde{G}^{-m}\left(t_{j}\right)\right\|_{(0, m)}\left\|\tilde{G}^{m}\left(t_{i}\right)\right\|_{(m, 0)}\left(\frac{C_{m} L_{m} T}{k}+1\right)^{j-i} .
\end{aligned}
$$

We rewrite $j-i=\lfloor r k / T\rfloor-\lfloor s k / T\rfloor \leq 1+\lfloor(r=s) k / T\rfloor=1+k\lfloor(r-s) k / T\rfloor / k$ to be able to introduce an exponential function in the limit $k \rightarrow \infty$ while $\lfloor(r-s) k / T\rfloor / k \rightarrow(r-s) / T$

$$
\begin{aligned}
\left(\frac{C_{m} L_{m} T}{k}+1\right)^{j-i} & \leq\left(\frac{C_{m} L_{m} T}{k}+1\right)\left(\left(\frac{C_{m} L_{m} T}{k}+1\right)^{k}\right)^{\lfloor(r-s) k / T\rfloor / k} \\
& \longrightarrow e^{C_{m} L_{m}(r-s)} \leq e^{C_{m} L_{m} T}
\end{aligned}
$$

This means that (26) goes to zero and the Cauchy sequence (25) must converge to a welldefined and bounded $U(t, s): D\left(A^{m}\right) \rightarrow D\left(A^{m-1}\right)$. In the lowest order $m=1$, the operators $U(t, s): D(A) \rightarrow X$ can then be continuously extended from the dense $D(A)$ to the whole space $X$ because they are clearly bounded on $X$ (as a combination of quasicontraction semigroup elements). To establish $U(t, s): D\left(A^{m}\right) \rightarrow D\left(A^{m}\right)$ bounded as the desired regularity result, we resort to Lemma 5 from Kato (1953) that states for reflexive Banach spaces that if a sequence $x_{k}$ converges weakly to $x$ and has $\left\{\left\|A x_{k}\right\|\right\}_{k}$ bounded, then $x \in D(A)$ and $A x$ is the weak limit of $A x_{k}$. [This result is actually missing in the proof in Penz (2016, Theorem 3.41, Theorem 3.42), where the boundedness was just directly inferred from the $U_{k}$ boundedness.] For this, we take $x=U(t, s) \varphi$ and $x_{k}=U_{k}(t, s) \varphi$ for any $\varphi \in D\left(A^{m}\right)$ and the Banach space $D\left(A^{m-1}\right)$ which is a reflexive Banach space by Lemma II.2. Then there is always a $f \in D\left(A^{m-1}\right)^{*}$ with dual norm 1 such that $(f, A x)=\|A x\|_{(m-1)}$ (Hahn-Banach theorem). This gives the estimate

$$
|(f, A x)| \leq\|A x\|_{(m-1)} .
$$

Now the weak limit $x=\mathrm{w}-\lim _{k \rightarrow \infty} x_{k}$ clearly follows as a result of even strong convergence in $D\left(A^{m-1}\right)$ and $\left\{\left\|A x_{k}\right\|_{(m-1)}\right\}_{k}$ bounded follows from $\left\{\left\|x_{k}\right\|_{(m)}\right\}_{k}$ bounded which was just shown in (29) above. Then the assertion of Lemma 5 in Kato (1953) says $A x=\mathrm{w}-\lim _{k \rightarrow \infty} A x_{k}$ from which follows

$$
\|A x\|_{(m-1)}=(f, A x)=\lim _{k \rightarrow \infty}\left(f, A x_{k}\right) \leq \limsup _{k \rightarrow \infty}\left\|A x_{k}\right\|_{(m-1)} .
$$

We first deal with the case $m=1$ which means for the inequality above

$$
\|A U(t, s) \varphi\| \leq \limsup _{k \rightarrow \infty}\left\|A U_{k}(t, s) \varphi\right\|
$$

and thus by introducing the $\|\cdot\|_{(1)}$ norm 


$$
\begin{aligned}
\|U(t, s) \varphi\|_{(1)} & =\|U(t, s) \varphi\|+\|A U(t, s) \varphi\| \leq\|U(t, s) \varphi\|+\limsup _{k \rightarrow \infty}\left\|A U_{k}(t, s) \varphi\right\| \\
& =\limsup _{k \rightarrow \infty}\left\|U_{k}(t, s) \varphi\right\|_{(1)}+\|U(t, s) \varphi\|-\limsup _{k \rightarrow \infty}\left\|U_{k}(t, s) \varphi\right\| .
\end{aligned}
$$

In the limit, the last two terms cancel because we already showed that $U_{k}(t, s) \rightarrow U(t, s)$ converges on $X$. So using the estimates from (29) and (30), we get

$$
\|U(t, s)\|_{(1,1)} \leq C_{1}^{\prime} \exp \left(C_{1} L_{1} T\right),
$$

where the additional constants from (29) have been collected in $C_{1}^{\prime}$. This means $U_{k}(t, s) \rightarrow U(t, s)$ converges also as a bounded operator $D(A) \rightarrow D(A)$. The next step is already for arbitrary $m$ and we use (32) again

$$
\begin{aligned}
\|U(t, s) \varphi\|_{(m)} & =\|U(t, s) \varphi\|+\left\|A^{m} U(t, s) \varphi\right\|=\|U(t, s) \varphi\|+\left\|A^{m-1} A U(t, s) \varphi\right\| \\
& =\|U(t, s) \varphi\|+\|A U(t, s) \varphi\|_{(m-1)}-\|A U(t, s) \varphi\| \\
& \leq\|U(t, s) \varphi\|+\underset{k \rightarrow \infty}{\limsup }\left\|A U_{k}(t, s) \varphi\right\|_{(m-1)}-\|A U(t, s) \varphi\| .
\end{aligned}
$$

Next the $D\left(A^{m-1}\right)$ norm gets rewritten to a $D\left(A^{m}\right)$ norm

$$
\begin{aligned}
\|U(t, s) \varphi\|_{(m)} & \leq \limsup _{k \rightarrow \infty}\left\|U_{k}(t, s) \varphi\right\|_{(m)} \\
& +\|U(t, s) \varphi\|-\limsup _{k \rightarrow \infty}\left\|U_{k}(t, s) \varphi\right\| \\
& -\|A U(t, s) \varphi\|+\limsup _{k \rightarrow \infty}\left\|A U_{k}(t, s) \varphi\right\| .
\end{aligned}
$$

This time the whole two last lines vanish in the limit because in the meantime we also established convergence on $D(A)$, so with the estimates from (29) and (30) we finally get boundedness $D\left(A^{m}\right) \rightarrow D\left(A^{m}\right)$

$$
\|U(t, s)\|_{(m, m)} \leq C_{m}^{\prime} \exp \left(C_{m} L_{m} T\right) .
$$

The evolution system properties (i) and (ii) from Definition IV.1 follow directly from the semigroup properties of the $U_{k}{ }^{(i)}$ and uniform convergence of $U_{k}(t, s)$ in $s, t$ which allows us to exchange limits. Finally we have to show that the evolution semigroup is a solution to the Cauchy problem $\partial_{t} U(t, s)$ $=G(t) U(t, s)$ (the $\partial_{s}$ version can be handled equivalently). Again we use uniform convergence and interchange time differentiation at $t \neq t_{i} \in P_{k}$ and the limit for the sequence $U_{k}(t, s)$

$$
\partial_{t} U(t, s)=\partial_{t} \lim _{k \rightarrow \infty} U_{k}(t, s)=\lim _{k \rightarrow \infty} \partial_{t} U_{k}(t, s)=\lim _{k \rightarrow \infty} G\left(\lfloor t\rfloor_{k}\right) U_{k}(t, s) .
$$

On $D(A)$, we have $U_{k}(t, s) \rightarrow U(t, s) \in \mathcal{B}(D(A), D(A))$ as well as $G\left(\lfloor t\rfloor_{k}\right) \rightarrow G(t) \in \mathcal{B}(D(A), X)$, so we can establish the limits independently and get the desired evolution system for the Cauchy problem $\partial_{t} U(t, s)=G(t) U(t, s)$. If $t=t_{i} \in P_{k}$, the right and left derivatives will differ and yield $G\left(t_{i}\right)$ and $G\left(t_{i-1}\right)$, respectively, but in the limit $k \rightarrow \infty$ they are equal again because of the assumed continuity of the generator $G$ in time.

Note IV.5. In Schmid and Griesemer (2016), it is assumed that $G(t)$ is the generator of a group instead of a semigroup to get solutions to the equation involving the time derivative $\partial_{t}$ instead of just the right derivative $\partial_{t}^{+}$.

Note IV.6. Establishing evolution systems between the different orders of graph-norm spaces $D\left(A^{k}\right)$ bears strong resemblance to the construction of the so-called "Sobolev towers" in Engel-Nagel (2000, II.5.a), although there the construction is only for time-independent generators.

Note IV.7. A similar proof strategy can be employed to show Fréchet differentiability of the solution to (1) in a Banach space including the time variable with respect to the perturbations $B$. See Penz (2016, Theorem 4.10) for such a result and Penz and Ruggenthaler (2015) for a similar result using the completely different proof method of "successive substitutions." 


\section{APPLICATION TO THE SCHRÖDINGER EQUATION}

To treat the quantum mechanical case of particles in singular Coulombic potentials and other unbounded potentials, we make use of the following lemma from Fourier analysis. Here the number of dimensions of the underlying space actually plays a crucial role and we are limited to the dimension $n \leq 3$ for the one-particle configuration space in all further results because of the following lemma.

Lemma V.1 (Reed-Simon II, 1975, Theorem IX.28). Let $\varphi \in W^{2,2}\left(\mathbb{R}^{n}\right), n \leq 3$. Then for all $\alpha>0$, there is a $\beta>0$ independent of $\varphi$ such that

$$
\|\varphi\|_{\infty} \leq \alpha\|\Delta \varphi\|_{2}+\beta\|\varphi\|_{2}
$$

The next theorem is then a standard application of Lemma V.1 together with the KatoRellich theorem to the case of the Schrödinger Hamiltonian with zero boundary conditions; see Reed-Simon II (1975, Theorem X.12) and Kato (1995, Theorem V.4.11). The Kato-Rellich theorem states that if $A$ is self-adjoint and $B$ symmetric, then $A+B$ is also self-adjoint whenever $B$ is $A$-bounded with the relative bound strictly smaller than 1 . The critical condition is thus that the potential turns out to be $\Delta$-bounded. The spatial domain $\Omega$ is always assumed to be a (open and connected) subset of $\mathbb{R}^{n}, n \leq 3$.

Theorem V.2 (Reed-Simon II, 1975, Theorem X.15). Given a real potential $v \in L^{2}\left(\mathbb{R}^{n}\right)$ $+L^{\infty}\left(\mathbb{R}^{n}\right), n \leq 3$, the Hamiltonian $-\Delta+v$ is self-adjoint on $W^{2,2}(\Omega) \cap W_{0}^{1,2}(\Omega)$.

Definition V.3. The space of Kato perturbations $L^{2}\left(\mathbb{R}^{n}\right)+L^{\infty}\left(\mathbb{R}^{n}\right)$ is equipped with the norm

$$
\|v\|_{2+\infty}=\inf \left\{\left\|v_{1}\right\|_{2}+\left\|v_{2}\right\|_{\infty} \mid v_{1} \in L^{2}\left(\mathbb{R}^{n}\right), v_{2} \in L^{\infty}\left(\mathbb{R}^{n}\right), v=v_{1}+v_{2}\right\} .
$$

The following notation for the extension of potentials to multi-particle systems with $N$ particles is borrowed from Lammert (2018). Note that in the published version of this work that we cite along the preprint, the respective notation has vanished again.

Definition V.4. For a one-point function $v: \Omega \rightarrow \mathbb{R}$, we define

$$
\Gamma v: \Omega^{N} \rightarrow \mathbb{R},\left(x_{1}, \ldots, x_{N}\right) \mapsto \sum_{i=1}^{N} v\left(x_{i}\right)
$$

and similarly for a two-point function $w: \Omega \times \Omega \rightarrow \mathbb{R}$

$$
\Gamma w: \Omega^{N} \rightarrow \mathbb{R},\left(x_{1}, \ldots, x_{N}\right) \mapsto \frac{1}{2} \sum_{i, j=1 i \neq j}^{N} w\left(x_{i}, x_{j}\right) .
$$

Lemma V.5. Given the potentials $v, v_{\text {int }} \in L^{2}\left(\mathbb{R}^{n}\right)+L^{\infty}\left(\mathbb{R}^{n}\right), n \leq 3$, and the interaction potential $w\left(x_{1}, x_{2}\right)=v_{\text {int }}\left(x_{1}-x_{2}\right)$, the multiplication operators $\Gamma v$ and $\Gamma w$ are both $\Delta$-bounded with the relative bound 0 . There is further a constant $\beta>0$ such that the following estimates hold for all $\varphi \in W^{2,2}\left(\Omega^{N}\right)$

$$
\begin{aligned}
\|(\Gamma v) \varphi\|_{2} & \leq N \beta\|v\|_{2+\infty}\|\varphi\|_{2,2}, \\
\|(\Gamma w) \varphi\|_{2} & \leq \frac{N(N-1)}{2} \beta\left\|v_{\text {int }}\right\|_{2+\infty}\|\varphi\|_{2,2} .
\end{aligned}
$$

Proof. We adopt the following notation for the norm of the Hilbert space $L^{2}(\Omega)$ where we assume all coordinates $x_{j \neq i}$ fixed and analogously if only one coordinate $x_{i}$ is fixed

$$
\|\varphi\|_{2}^{(i)}=\left(\int_{\Omega}|\varphi|^{2} \mathrm{~d} x_{i}\right)^{1 / 2}, \quad\|\varphi\|_{2}^{(j \neq i)}=\left(\int_{\Omega}|\varphi|^{2} \mathrm{~d} x_{j \neq i}\right)^{1 / 2} .
$$


Note that it holds $\|\varphi\|_{2}=\|\| \varphi\left\|_{2}^{(i)}\right\|_{2}^{(j \neq i)}$ so that we have

$$
\|(\Gamma v) \varphi\|_{2} \leq \sum_{i=1}^{N}\left\|v\left(x_{i}\right) \varphi\right\|_{2}=\sum_{i=1}^{N}\|\| v\left(x_{i}\right) \varphi\left\|_{2}^{(i)}\right\|_{2}^{(j \neq i)} .
$$

Now the inner norm is estimated with the decomposition $v=v_{1}+v_{2}, v_{1} \in L^{2}, v_{2} \in L^{\infty}$ as $\left\|v\left(x_{i}\right) \varphi\right\|_{2}^{(i)}$ $\leq\left\|v_{1}\right\|_{2}\|\varphi\|_{\infty}^{(i)}+\left\|v_{2}\right\|_{\infty}\|\varphi\|_{2}^{(i)}$, where we use the obvious notation of $\|\varphi\|_{\infty}^{(i)}$ as the essential supremum of $\varphi$ over all $x_{i} \in \Omega$. Note that $\left\|v_{1}\right\|_{2}$ and $\left\|v_{2}\right\|_{\infty}$ are just numbers with no free variables left. It is now time to invoke Lemma V.1 and have for arbitrarily small $\alpha_{i}>0$

$$
\|\varphi\|_{\infty}^{(i)} \leq \alpha_{i}\left\|\Delta_{i} \varphi\right\|_{2}^{(i)}+\beta_{i}\|\varphi\|_{2}^{(i)} .
$$

Combination of these estimates gives

$$
\|(\Gamma v) \varphi\|_{2} \leq \sum_{i=1}^{N}\left(\alpha_{i}\left\|v_{1}\right\|_{2}\left\|\Delta_{i} \varphi\right\|_{2}+\left(\beta_{i}\left\|v_{1}\right\|_{2}+\left\|v_{2}\right\|_{\infty}\right)\|\varphi\|_{2}\right) .
$$

A final trick is needed to have the full Laplacian $\Delta$ instead of $\Delta_{i}$ only involving $x_{i}$. For this, we observe that by moving to the Fourier domain with coordinates $k_{i} \in \mathbb{R}^{n}$

$$
\left\|\Delta_{i} \varphi\right\|_{2}=\left\|k_{i}^{2} \hat{\varphi}\right\|_{2} \leq\left\|\sum_{j=1}^{N} k_{j}^{2} \hat{\varphi}\right\|_{2}=\|\Delta \varphi\|_{2} .
$$

Now define $\alpha=\max _{i} \alpha_{i}$ (but still arbitrarily small) and $\beta=\max \left\{\beta_{1}, \ldots, \beta_{N}, \alpha, 1\right\}$ and we get

$$
\|(\Gamma v) \varphi\|_{2} \leq N \alpha\left\|v_{1}\right\|_{2}\|\Delta \varphi\|_{2}+N\left(\beta\left\|v_{1}\right\|_{2}+\left\|v_{2}\right\|_{\infty}\right)\|\varphi\|_{2} .
$$

This means $\Gamma v$ is $\Delta$-bounded with the relative bound 0 . If we further introduce $\|v\|_{2+\infty}$ and choose $v_{1}$ and $v_{2}$ accordingly, then with $\beta$ defined as above we can take it out as an upper estimate. Together with the equivalence of the graph norm of $D(\Delta)$ and the Sobolev norm $\|\cdot\|_{2,2}$, we arrive at the desired

$$
\|(\Gamma v) \varphi\|_{2} \leq N \beta\|v\|_{2+\infty}\|\varphi\|_{2,2} .
$$

The proof for the two-point potential that is defined as an interaction potential involving $v_{\text {int }}\left(x_{i}-x_{j}\right)$ is analogous but one first has to rotate the whole $\Omega^{N} \subseteq \mathbb{R}^{n N}$ so that $x_{i}-x_{j}$ matches the $x_{1}$ coordinate. This is possible invariantly because the $L^{2}$-norm is a rotational invariant. The rest of the proof stays the same; we only consider $N(N-1) / 2$ components in the sum instead of only $N$.

Note V.6. The Lemma V.5 above allows for an extension of Theorem V.2 to multi-particle systems with Hamiltonian $H=-\Delta+\Gamma w+\Gamma v$ if the involved potentials are of type $v, v_{\text {int }} \in L^{2}\left(\mathbb{R}^{n}\right)+L^{\infty}\left(\mathbb{R}^{n}\right)$ with $w\left(x_{i}, x_{j}\right)=v_{\text {int }}\left(x_{i}-x_{j}\right)$. The proof structure of Lemma V.5 was inspired by a theorem with this assertion given in Reed-Simon II (1975, Theorem X.16).

Definition V.7. We extend Definition V.3 (Kato perturbations) to Sobolev-Kato perturbations, defined as the space of potentials

$$
W^{m, 2+\infty}\left(\mathbb{R}^{n}\right)=\left\{v\left|D^{\alpha} v \in L^{2}\left(\mathbb{R}^{n}\right)+L^{\infty}\left(\mathbb{R}^{n}\right),\right| \alpha \mid \leq m\right\}
$$

with norm

$$
\|v\|_{m, 2+\infty}=\sum_{|\alpha| \leq m}\left\|D^{\alpha} v\right\|_{2+\infty}
$$

Lemma V.8. Given the potentials $v, v_{\text {int }} \in W^{2 m, 2+\infty}\left(\mathbb{R}^{n}\right), n \leq 3$, and $w\left(x_{1}, x_{2}\right)=v_{\text {int }}\left(x_{1}-x_{2}\right)$, the multiplication operators $\Gamma v$ and $\Gamma w$ are both $\Delta$-bounded of order $m+1$ with the relative bound 0 . There is further a constant $\beta>0$ such that the following estimates hold for all $\varphi \in W^{2(m+1), 2}\left(\Omega^{N}\right)$ :

$$
\begin{aligned}
\|(\Gamma v) \varphi\|_{2 m, 2} \leq N \beta\|v\|_{2 m, 2+\infty}\|\varphi\|_{2(m+1), 2}, \\
\|(\Gamma w) \varphi\|_{2 m, 2} \leq \frac{N(N-1)}{2} \beta\left\|v_{\text {int }}\right\|_{2 m, 2+\infty}\|\varphi\|_{2(m+1), 2} .
\end{aligned}
$$


Proof. Lemma V.5 already shows the case $m=0$ and proceeding from that we give the proof for arbitrary orders $m$. We start by writing out the involved Sobolev space norm explicitly, then employ the general Leibniz rule for multivariable calculus

$$
\begin{aligned}
\|(\Gamma v) \varphi\|_{2 m, 2} \sim \sum_{|\alpha| \leq 2 m}\left\|D^{\alpha}((\Gamma v) \varphi)\right\|_{2} & =\sum_{|\alpha| \leq 2 m}\left\|\sum_{v \leq \alpha}\left(\begin{array}{c}
\alpha \\
v
\end{array}\right)\left(D^{v}(\Gamma v)\right) D^{\alpha-v} \varphi\right\|_{2} \\
& \leq\left(\begin{array}{c}
2 m \\
m
\end{array}\right) \sum_{|\alpha| \leq 2 m} \sum_{v \leq \alpha}\left\|\left(D^{v}(\Gamma v)\right) D^{\alpha-v} \varphi\right\|_{2} .
\end{aligned}
$$

The multi-index binomial coefficient is estimated by its largest possible value. Next we use the property of $\Gamma v$ that makes the potential the sum of one-coordinate potentials. Thus instead of the full $D^{v}$ only $D^{v_{i}}$ acts on the individual terms of the sum in $\Gamma v$. Note that these $v_{i}$ from $v=\left(v_{1}, \ldots, v_{N}\right)$ are still $n$-tuples. In any case, we have

$$
D^{v}(\Gamma v)=\sum_{i=1}^{N} D^{v_{i}} v\left(x_{i}\right)
$$

and thus

$$
\begin{aligned}
\sum_{|\alpha| \leq 2 m}\left\|D^{\alpha}((\Gamma v) \varphi)\right\|_{2} & \leq\left(\begin{array}{c}
2 m \\
m
\end{array}\right) \sum_{|\alpha| \leq 2 m} \sum_{\nu \leq \alpha} \sum_{i=1}^{N}\left\|\left(D^{\nu_{i}} v\left(x_{i}\right)\right) D^{\alpha-v} \varphi\right\|_{2} \\
& =\left(\begin{array}{c}
2 m \\
m
\end{array}\right) \sum_{|\alpha| \leq 2 m} \sum_{\nu \leq \alpha} \sum_{i=1}^{N}\|\|\left(D^{\nu_{i}} v\left(x_{i}\right)\right) D^{\alpha-v} \varphi\left\|_{2}^{(i)}\right\|_{2}^{(j \neq i)}
\end{aligned}
$$

like in (47). The proof then proceeds exactly like in Lemma V.5 since we have $D^{v_{i}} v\left(x_{i}\right) \in L^{2}+L^{\infty}$ due to the assumption $v \in W^{m, 2+\infty}$. In total, we get the estimate

$$
\sum_{|\alpha| \leq 2 m}\left\|D^{\alpha}((\Gamma v) \varphi)\right\|_{2} \leq N \beta^{\prime}\left(\begin{array}{c}
2 m \\
m
\end{array}\right)\|v\|_{2 m, 2+\infty}\|\varphi\|_{2(m+1), 2},
$$

where the sums over the multi-indices get combined and estimated by the higher Sobolev norms. The order of the Sobolev norm of $\varphi$ has increased by 2 because we had to rely on Lemma V.1 again. The constant $\beta^{\prime}$ that is defined similar as in lemma V.5 before gets combined together with the binomial coefficient to form a constant $\beta$ and we arrive at the desired result. If we keep the arbitrarily small $\alpha_{i}$ that are introduced analogously to (48), then this also yields the desired $\Delta$-boundedness of order $m+1$ with the relative bound 0 . In both cases, the equivalence of the graph norm of $D\left(\Delta^{m}\right)$ and the Sobolev norm $\|\cdot\|_{2 m, m}$ gets applied.

The way for a two-point potential is the same as before with the only difference that we have to observe

$$
D^{v}(\Gamma w)=\frac{1}{2} \sum_{\substack{i, j=1 \\ i \neq j}} D^{v_{i}} D^{v_{j}} v_{\text {int }}\left(x_{i}-x_{j}\right)
$$

before rotating $x_{i}-x_{j}$ again so that it matches the $x_{1}$ coordinate in the individual contributions of the norm.

Theorem V.9. The $N$-particle Schrödinger equation

$$
\mathrm{i} \partial_{t} \psi(t)=H(t) \psi(t)=(-\Delta+\Gamma w+\Gamma v(t)) \psi(t)
$$

on the Hilbert space $L^{2}\left(\Omega^{N}\right), \Omega \subseteq \mathbb{R}^{n}$ open and connected, $n \leq 3$, with

$$
v \in \operatorname{Lip}\left([0, T], W^{2(m-1), 2+\infty}\left(\mathbb{R}^{n}\right)\right)
$$

and $w\left(x_{i}, x_{j}\right)=v_{\text {int }}\left(x_{i}-x_{j}\right), v_{\mathrm{int}} \in W^{2(m-1), 2+\infty}\left(\mathbb{R}^{n}\right)$ has a well-defined unitary evolution system that is bounded as a mapping 


$$
U(t, s): W^{2 m, 2}\left(\Omega^{N}\right) \cap W_{0}^{m, 2}\left(\Omega^{N}\right) \rightarrow W^{2 m, 2}\left(\Omega^{N}\right) \cap W_{0}^{m, 2}\left(\Omega^{N}\right), \quad t, s \in[0, T] .
$$

This effectively establishes Sobolev regularity of solutions up to order $W^{2 m, 2}$.

Proof. We rewrite the Schrödinger equation as

$$
\partial_{t} \psi(t)=-\mathrm{i} H(t) \psi(t)=\mathrm{i}(\Delta-\Gamma w-\Gamma v(t)) \psi(t)
$$

and take $A=\mathrm{i} \Delta, G(t)=-\mathrm{i} H(t)$. Both operators are generators of contraction semigroups because of self-adjointness (Engel and Nagel, 2000, Theorem II.3.24, Stone theorem) in conjunction with Lemma V.5 and the Kato-Rellich theorem. Lemma V.8 tells us that $\Gamma v(t)$ and $\Gamma w$ are bounded operators $W^{2 m, 2}\left(\Omega^{N}\right) \rightarrow W^{2(m-1), 2}\left(\Omega^{N}\right)$ as well as $\Delta$-bounded of order $m$ with relative bound 0 . Thus all the requirements on the non-autonomous perturbation from Lemma III.4 are fulfilled and Theorem IV.4 becomes applicable which establishes the desired regularity result.

Note V.10. A final note shall make the setting even more "physical" and turns attention towards the standard example for external potentials and interactions, the Coulomb potential on $\Omega=\mathbb{R}^{3}$. If we take $v(x)=-|x|^{-1}$ (attractive) or $v_{\text {int }}(x)=|x|^{-1}$ (repulsive) those potentials lie in the class of Kato perturbations $L^{2}\left(\mathbb{R}^{3}\right)+L^{\infty}\left(\mathbb{R}^{3}\right)$. Even the more singular choice of $|x|^{-3 / 2+\varepsilon}$ for arbitrarily small $\varepsilon$ is permitted. Furthermore the potentials can be time-dependent under the constraint of the introduced Lipschitz condition. But those potentials already drop out of the next higher regularity class $W^{1,2+\infty}\left(\mathbb{R}^{3}\right)$ thus Theorem V.9 only guarantees Sobolev regularity up to $W^{2,2}$ for solutions to the Schrödinger equation with Coulomb potentials.

\section{ACKNOWLEDGMENTS}

With special thanks to Jonas Lampart for pointing out the studies of Schmid-Griesemer, as well as to Michael Ruggenthaler and Eric Stachura for helpful discussions. An anonymous referee spotted a critical flaw in the Proof of Lemma III.4, that in the course led to the new Definition III.1 and Lemma III.3, and helped to improve the manuscript substantially. This work was supported by the Erwin Schrödinger Fellowship No. J 4107-N27 of the FWF (Austrian Science Fund).

Bourgain, J., "On growth of Ssobolev norms in linear Schrödinger equations with smooth time dependent potential," J. Anal. Math. 77, 315-348 (1999).

Delort, J.-M., "Growth of Sobolev norms of solutions of linear Schrödinger equations on some compact manifolds," Int. Math. Res. Not. 2010(12), 2305-2328.

Engel, K.-J. and Nagel, R., One-Parameter Semigroups for Linear Evolution Equations (Springer, 2000).

Fournais, S., Lampart, J., Lewin, M., and Østergaard Sørensen, T., "Coulomb potentials and Taylor expansions in timedependent density functional theory," Phys. Rev. A 93, 062510 (2016).

Kato, T., "Integration of the equation of evolution in a Banach space," J. Math. Soc. J. 5, 208 (1953).

Kato, T., Perturbation Theory for Linear Operators (Springer, 1995).

Lammert, P. E., "In search of the Hohenberg-Kohn theorem," J. Math. Phys. 59, 042110 (2018); preprint arXiv:1412.3876v3. A Gamut of Hohenberg-Kohn Properties (2016).

Pazy, A., Semigroups of Linear Operators and Applications to Partial Differential Equations (Springer, 1983).

Penz M., "The density-potential mapping in quantum dynamics (doctoral thesis)," e-print arXiv:1610.05552 (2016).

Penz, M. and Ruggenthaler, M., "Functional differentiability in time-dependent quantum mechanics," J. Chem. Phys. 142, 124113 (2015).

Reed, M. and Simon, B., Methods of Modern Mathematical Physics, Vol. II: Fourier Analysis, Self-Adjointness (Academic Press, 1975).

Renardy, M. and Rogers, R. C., An Introduction to Partial Differential Equations, 2nd ed. (Springer, 2004).

Ruggenthaler, M., Penz, M., and van Leeuwen, R., "Existence, uniqueness, and construction of the density-potential mapping in time-dependent density-functional theory," J. Phys.: Condens. Matter 27, 203202 (2015).

Schmid, J. and Griesemer, M., "Kato's theorem on the integration of non-autonomous linear evolution equations," Math. Phys. Anal. Geom. 17, 265 (2014); preprint arXiv:1203.4700v3.

Schmid, J. and Griesemer, M., "Well-posedness of non-autonomous linear evolution equations in uniformly convex spaces," Math. Nachr. 290, 435 (2016); preprint arXiv:1501.06092v2. 